\title{
Anaerobic Threshold Biophysical Characterisation of the Four Swimming Techniques
}

\author{
Authors \\ Diogo Duarte Carvalho1, 2, Susana Soares ${ }^{1}$, Rodrigo Zacca1, 2, 3, João Sousa ${ }^{1}$, Daniel Almeida Marinho ${ }^{4,5}$, António José \\ Silva $^{5,6}$, João Paulo Vilas-Boas ${ }^{1,2}$, Ricardo J. Fernandes ${ }^{1,2}$
}

\section{Affiliations}

1 Faculty of Sport, Centre of Research, Education, Innovation and Intervention in Sport, University of Porto, Porto, Portugal

2 Porto Biomechanics Laboratory, University of Porto, Porto, Portugal

3 CAPES Foundation, Ministry of Education of Brazil, Brasília, Brazil

4 Department of Sport Sciences, University of Beira Interior, Covilhã, Portugal

5 Research Center in Sports Sciences, Health Sciences and Human Development, University of Trás-os-Montes and Alto Douro, Vila Real, Portugal

6 Department of Sport Sciences, University of Trás-osMontes and Alto Douro, Vila Real, Portugal

\section{Key words}

physiology, biomechanics, biophysics, anaerobic threshold, elite swimmers

accepted $\quad 07.07 .2019$

\section{Bibliography}

DOI https://doi.org/10.1055/a-0975-9532

Published online: 2020

Int J Sports Med

(C) Georg Thieme Verlag KG Stuttgart · New York

ISSN 0172-4622

\section{Correspondence}

Diogo Duarte Carvalho

Swimming

Faculty of Sport

University of Porto

4200-450 Porto

Portugal

Tel.: + 351/22/04 25 200, Fax: + 351/225/500 687

121101075@fade.up.pt

\begin{abstract}
The anaerobic threshold (AnT) seems to be not only a physiologic boundary but also a transition after which swimmers technique changes, modifying their biomechanical behaviour. We expanded the AnT concept to a biophysical construct in the four conventional swimming techniques. Seventy-two elite swimmers performed a $5 \times 200 \mathrm{~m}$ incremental protocol in their preferred swimming technique (with a $0.05 \mathrm{~m} \cdot \mathrm{s}^{-1}$ increase and a $30 \mathrm{~s}$ interval between steps). A capillary blood samples were collected from the fingertip and stroke rate (SR) and length (SL) determined for the assessment of [La], SR and SL vs. velocity inflexion points (using the interception of a pair of linear and exponential regression curves). The [La] values at the AnT were $3.3 \pm 1.0,3.9 \pm 1.1,2.9 \pm 1.34$ and $4.5 \pm 1.4 \mathrm{mmol} \cdot \mathrm{I}^{-1}$ (mean \pm SD) for front crawl, backstroke, breaststroke and butterfly, and its corresponding velocity correlated highly with those at SR and SL inflection points $(r=0.91-0.99, p<0.001)$. The agreement analyses confirmed that AnT represents a biophysical boundary in the four competitive swimming techniques and can be determined individually using [La] and/or SR/SL. Blood lactate increase speed can help characterise swimmers' anaerobic behaviour after AnT and between competitive swimming techniques.
\end{abstract}

\section{Introduction}

In swimming, competitive success can be compromised by high volume and indiscriminate training loads. Indeed, accurate and dedicated exercise prescription is required, reinforcing the impor- tance of regular training control and pool-based testing of biophysical measures [1-3]. From the complex group of swimming determinants, the physiological variables have been studied extensively using established testing procedures [e.g., [4]]. The most field-ap- 
plicable procedure is the anaerobic threshold concept $[\mathrm{AnT} ;[5,6]]$ allowing determination of the boundary after which the rise of swimming velocity leads to the loss of body homeostasis, as aerobic metabolism alone does not support energy requirements [7].

AnT can be assessed as the break point on the blood lactate concentration ([La]) vs. workload curve during incremental swimming $[5,8]$, identifying the individual exercise intensity after which lactate production exceeds its reduction, and swimming velocity is no longer sustainable for a long time period $[6,9,10]$. Occurring in the transition from moderate to heavy exercise intensity domains $[11,12]$, AnT is considered as one of the most consistent predictors of performance in cyclic and individual sports, particularly in swimming $[9,10,13]$. However, the fixed [La] $4 \mathrm{mmol} \cdot \mathrm{I}^{-1}$ value proposed by Mader et al. (1978) does not consider inter-individual differences, overestimating the real aerobic capacity of aerobically trained athletes $[7,8,14]$.

Biomechanics is of growing importance in swimming training diagnosis, leading to a biophysical approach to training prescription and evaluation. In fact, combining the physiologic and biomechanical areas of expertise allows better control of the complexity of training loads and increase swimming performance $[12,15]$. Some studies already reported technical changes as swimming intensity rises, particularly identifying a stroke rate (SR) increase and stroke length (SL) deterioration after the AnT $[5,15]$. Therefore, AnT is purportedly not only a physiologic boundary, but also decisively influences the behaviour of biomechanical and coordinative variables $[11,17]$. However, probably due to the complexity of the motor task and constraints of the aquatic environment, these findings were only evident for front crawl swimming (with backstroke, breaststroke and butterfly being negatively discriminated) and using samples close to 10 subjects.

Our main aim was to confirm the AnT as the boundary above which [La], SR and SL exhibit significant changes. This will be done for the four conventional swimming techniques and using a representative cohort of trained swimmers using adequately powered sample size. We hypothesized that [La] at AnT ([La $]_{\text {AnT }}$ ) in front crawl and backstroke will be lower than the standard $4 \mathrm{mmol} \cdot \mathrm{I}^{-1}$ value (leading to relevant practical differences between corresponding swimming velocities) and that in breaststroke and butterfly it will be higher than that fixed value (as they are less economical swimming techniques) [18]. It is also expected that SR will rise and SL decrease after the AnT intensity due to changes in coordination strategies. Complementary, given that the anaerobic energy systems contribute substantially to short duration swimming events (and the majority of competitions lasts less than $2 \mathrm{~min}$ ), we have also assessed the swimmers' anaerobic behaviour in different techniques [12].

\section{Materials and Methods}

Seventy-two international level swimmers (47 males and 25 females), specialists in front crawl $(n=38)$, backstroke $(n=15)$, butterfly $(n=11)$ and breaststroke $(n=8)$, volunteered to participate. Their main physical characteristics were: $20.3 \pm 3.1$ vs. $18.4 \pm 2.5 \mathrm{y}$, $73.9 \pm 7.3$ vs. $58.9 \pm 5.7 \mathrm{~kg}$ body mass, $1.80 \pm 0.06 \mathrm{vs} .1 .70 \pm 0.04 \mathrm{~m}$ height, $8.0 \pm 2.5$ vs. $16.0 \pm 5.7$ percentage of fat mass and $811 \pm 32$ vs. $808 \pm 39$ FINA points of best competitive performance for male and female swimmers (respectively). Swimmers were informed about the purpose of the evaluations and provided individual consent for participation in accordance with this journal [19], the local research ethics committee (code $n^{\circ}$ CEFADE 28 2019) and the Declaration of Helsinki.

In a $25 \mathrm{~m}$ indoor pool with water temperature of $27^{\circ} \mathrm{C}$, after an $800 \mathrm{~m}$ standardised low intensity warm-up, each subject performed a $5 \times 200 \mathrm{~m}$ intermittent incremental protocol, with $0.05 \mathrm{~m} \cdot \mathrm{s}^{-1}$ increase per step and a $30 \mathrm{~s}$ rest interval $[11,12,20]$. In-water starts were used and initial velocities were established by the swimmer's current individual $400 \mathrm{~m}$ performance less five velocity increments (first step: velocity at $400 \mathrm{~m}-0.20 \mathrm{~m} \cdot \mathrm{s}^{-1}$ ). Performance was measured using a Seiko stopwatch and controlled through auditory signals. Each step represented the same relative intensity for all swimmers, independent of the swimming technique, who were verbally encouraged to reach their maximal exertion during the last $200 \mathrm{~m}$ step.

Capillary blood samples for [La] analysis were collected from the fingertip at rest, in the intervals between steps and 1, 3, 5 and 7 min after the protocol using a Lactate Pro 2 analyser (Arkay, Inc, Kyoto, Japan) [12]. SR was determined as the number of cycles per min (using a Seiko stopwatch with a base 3 upper limb cycles frequency meter function) and SL was calculated by dividing the mean velocity by SR (both were assessed as the mean value of each $50 \mathrm{~m}$ per $200 \mathrm{~m}$ step) [20]. By computing the [La], SR, and SL vs. velocity, linear and exponential curves were determined using the least square method $[8,17]$. After a visual inspection of the plots, and considering the respective residue value, the inflection points were assumed to be the intersection at the maximal fit situation, of a combined pair of linear and exponential regressions. In addition, linear inter- or extrapolation of the [La]/velocity curve allowed the determination of v4 and v3.5 [20], as displayed ( $\triangleright$ Fig. 1).

Each swimmer's anaerobic profile was established with two derived indicators: (i) the blood lactate increase rate ([La $]_{\text {rate; }}$ adapted from [21]), as the ratio between the net [La] and velocities ( $\Delta[\mathrm{La}]$ and $\Delta v$, respectively) of the two last protocol steps; and (ii) the blood lactate increase speed (BLIS) of $4^{\text {th }}$ and $5^{\text {th }}$ steps, as the time rate of net [La] per step (difference between the [La] obtained after the current step and the previous one, and the time needed to accomplish the $200 \mathrm{~m}$ step; adapted from [1]). Swimming techniques were analysed individually and by alternated (front crawl and backstroke) and simultaneous (breaststroke and butterfly) upper and lower limb-action groups, given the obvious dichotomy between them $[4,18]$.

Mean and standard deviation of measures were computed, and data were checked for distribution normality with the Shapiro-Wilk test. One-way independent and repeated measures ANOVA were performed to compare velocities, [La], SR and SL between swimming techniques and along the incremental protocol. Where a significant F-value was obtained, a Bonferroni post hoc was conducted to identify the pairwise differences and partial eta-squared $\left(\eta^{2}\right)$ used for computing the effect size. A paired samples Student's $t$-test was used to assess the differences between $v_{A n T}$ and $v_{4}, v_{3.5}$,

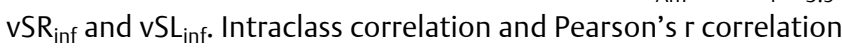
coefficients quantified the degree of reliability and association between $v_{\text {AnT }}$, vSR inf $_{\text {and }} v \mathrm{SL}_{\text {inf }}$ (respectively) [22]. Agreement analysis between the inflexion points was also performed (using $v_{A n T}$ as the reference) through both Passing-Bablok [23] regression and 


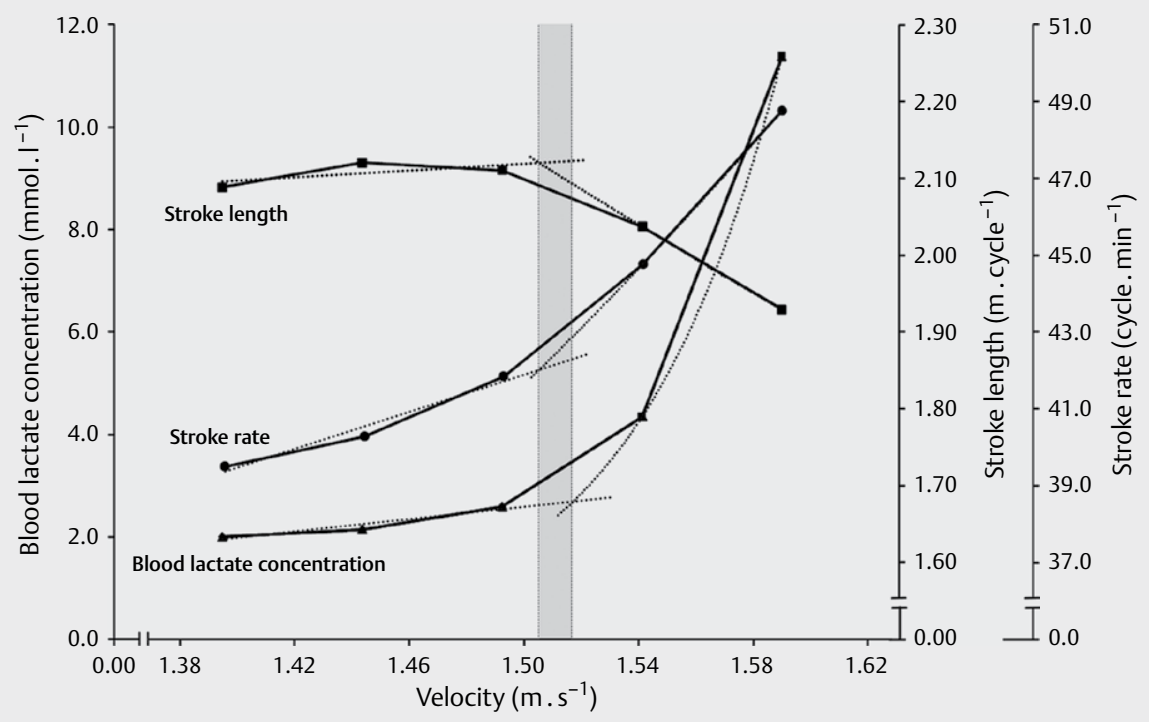

- Fig. 1 Determination of the inflection points of blood lactate concentrations, stroke rate and stroke length vs. velocity of one swimmer.

Bland-Altman [24] plot analysis (MedCalc Software, version 17.3, Mariakerke, Belgium) to assess the absence of systematic differences between measurements $(\rho \leq 0.05)$.

\section{Results}

In $>$ Fig. 2, the [La] vs. velocity curves are presented for each conventional swimming technique, displaying similar shapes although with diverse values. Velocity of $5^{\text {th }}$ step corresponded to $\sim 96 \%$ of the swimmers $200 \mathrm{~m}$ best performance and $99-100 \%$ of $400 \mathrm{~m}$ front crawl best performance. Increases in swimming velocity along the $5 \times 200$ m protocol in all swimming techniques $\left(p<0.001 ; \eta^{2}=0.88-\right.$ 0.95 ) implied, between first and last step of $200 \mathrm{~m}$, higher [La] (front crawl 288-845\%; backstroke 162-700\%; butterfly 163-650\%; breaststroke 193-910\%) higher SR (front crawl 112-170\%; backstroke 125-241\%; butterfly 115-170\% ; breaststroke 119-207\%), and lower SL (front crawl 1-14\%; backstroke 8-36\%; butterfly 1-22\%; breaststroke 5-30\% ), all for $\rho<0.001$ and $\eta^{2}=0.83-0.99$.

When analysing alternated and simultaneous upper and lower limb-action swimming techniques, we observed the same biophysical behaviour, i.e., the rise in swimming velocity $\left(F_{4.208}=219.66\right.$, $\eta^{2}=0.95$ vs. $\left.F_{4.76}=25.71, \eta^{2}=0.87\right)$ implied, between first and last step of $200 \mathrm{~m}$, higher [La] $\left(\mathrm{F}_{4.208}=133.06, \eta^{2}=0.92 \mathrm{vs}\right.$. $\mathrm{F}_{4.76}=71.63$, $\left.\eta^{2}=0.95\right)$ higher $S R\left(F_{4.208}=118.27 \eta^{2}=0.91\right.$; and $F_{4.76}=40.52$, $\left.\eta^{2}=0.91\right)$, and lower $S L\left(F_{4.208}=68.08, \eta^{2}=0.85\right.$; and $F_{4.76}=16.07$, $\eta^{2}=0.80$ ), all for $\rho<0.001$. The evolution of SR and SL along the incremental protocol for all analysed swimming techniques can be observed in $>$ Fig. 3.

- Table 1 presents mean and SD of [La $]_{A n T}, \mathrm{v}_{\mathrm{AnT}}, \mathrm{VSR}_{\text {inf }}, \mathrm{VSL}_{\mathrm{inf}}, \mathrm{V}_{3.5}, \mathrm{v}_{4}$, $\left[\mathrm{La}^{-}\right]_{\text {rate }}$ and $4^{\text {th }}$ and $5^{\text {th }}$ BLIS for the four conventional swimming techniques and the alternated and simultaneous techniques. [La $]_{\text {AnT }}$ and [La] $]_{\text {rate }}$ ranged from 1.3 to $5.6 \mathrm{mmol} \cdot \mathrm{I}^{-1}$ and 28 to $258\left(\mathrm{mmol} \cdot \mathrm{I}^{-1}\right)$. $\left(\mathrm{m} \cdot \mathrm{s}^{-1}\right)$ in front crawl, 1.5 to $5.4 \mathrm{mmol} \cdot \mathrm{I}^{-1}$ and 18.6 to 193.3 $\left(\mathrm{mmol} \cdot \mathrm{I}^{-1}\right) \cdot\left(\mathrm{m} \cdot \mathrm{s}^{-1}\right)$ in backstroke, 2.5 to $6.8 \mathrm{mmol} \cdot \mathrm{I}^{-1}$ and 37.6 to $266.7\left(\mathrm{mmol} \cdot \mathrm{I}^{-1}\right) \cdot\left(\mathrm{m} \cdot \mathrm{s}^{-1}\right)$ in butterfly and 1.8 to $5.4 \mathrm{mmol} \cdot \mathrm{I}^{-1}$ and 60.4 to $102.9\left(\mathrm{mmol} \cdot \mathrm{l}^{-1}\right) \cdot\left(\mathrm{m} \cdot \mathrm{s}^{-1}\right)$ in breaststroke, exhibiting highly interindividual variability.

When the swimming techniques were analysed individually, results showed that: (i) $v_{\text {AnT }}$ was lower than $v_{4}$ (but similar to the $v_{3.5}$ ) in front crawl; (ii) $v_{\text {AnT }}$ was similar to the $v_{4}$ (but higher than the $v_{3.5}$ ) in backstroke and butterfly; and (iii) $\mathrm{v}_{\mathrm{AnT}}$ was not different from both $\mathrm{v}_{4}$ and $\mathrm{v}_{3.5}$ in breaststroke. When analysed between groups, results showed that: (i) $v_{A n T}$ was lower than $v_{4}$ and $v_{3.5}$ in alternated swimming techniques; and (ii) $\mathrm{v}_{\mathrm{AnT}}$ was similar to $\mathrm{v}_{4}$ (but higher than $\left.v_{3.5}\right)$ ) in simultaneous techniques. In addition, the four swimming techniques $\mathrm{v}_{\text {AnT }}$ corresponded to $\sim 92 \%$ of v200 at the $5^{\text {th }}$ step $(92 \pm 3,93 \pm 3,93 \pm 4$ and $92 \pm 3 \%$, for front crawl, backstroke, butterfly and breaststroke, respectively), with front crawl presenting higher $v_{\text {AnT }}$ than butterfly, and breaststroke the lowest $v_{\text {AnT }}$ value.

Analyses of both physiological and biomechanical inflection points showed that the $\mathrm{v}_{\mathrm{AnT}}, \mathrm{vSR}_{\text {inf }}$ and $v \mathrm{vL}_{\text {inf }}$ were coincident for front crawl, backstroke, butterfly and breaststroke. In all the swimming techniques, intraclass correlation between physiological and biomechanical inflection points was excellent (ICC: 0.947 to 0.996 ; $95 \% \mathrm{Cl}: 0.861$ to 0.998$), v_{\text {AnT }}$ showed a strong direct relationship with vSR inf $_{\text {( }}$ : 0.948 to 0.995 ) and $v S L_{\text {inf }}$ ( $r: 0.953$ to 0.995 ), all for $\rho<0.001$. Passing-Block regressions between physiological and biomechanical inflection points are presented in - Fig. 4, the $95 \%$ confidence intervals of the slope and intercept included or were very close to 1 and zero, respectively. Similar results were observed in Bland-Altman plot reports ( $>$ Fig. 5), where estimations were almost unbiased and between the $95 \%$ limits of agreement. In addition, the slope of the Bland Altman regression showed evidence of a proportional error in the $\mathrm{v}_{\mathrm{AnT}} \mathrm{VS}$. $\mathrm{VSR}_{\text {inf }}$ comparison in the breaststroke technique.

In relation to swimmers' anaerobic characteristics, we observed that: (i) [La $]_{\text {rate }}$ is similar in all four swimming techniques; (ii) BLIS is higher in the $5^{\text {th }} 200 \mathrm{~m}$ step for all swimming techniques; (iii) 

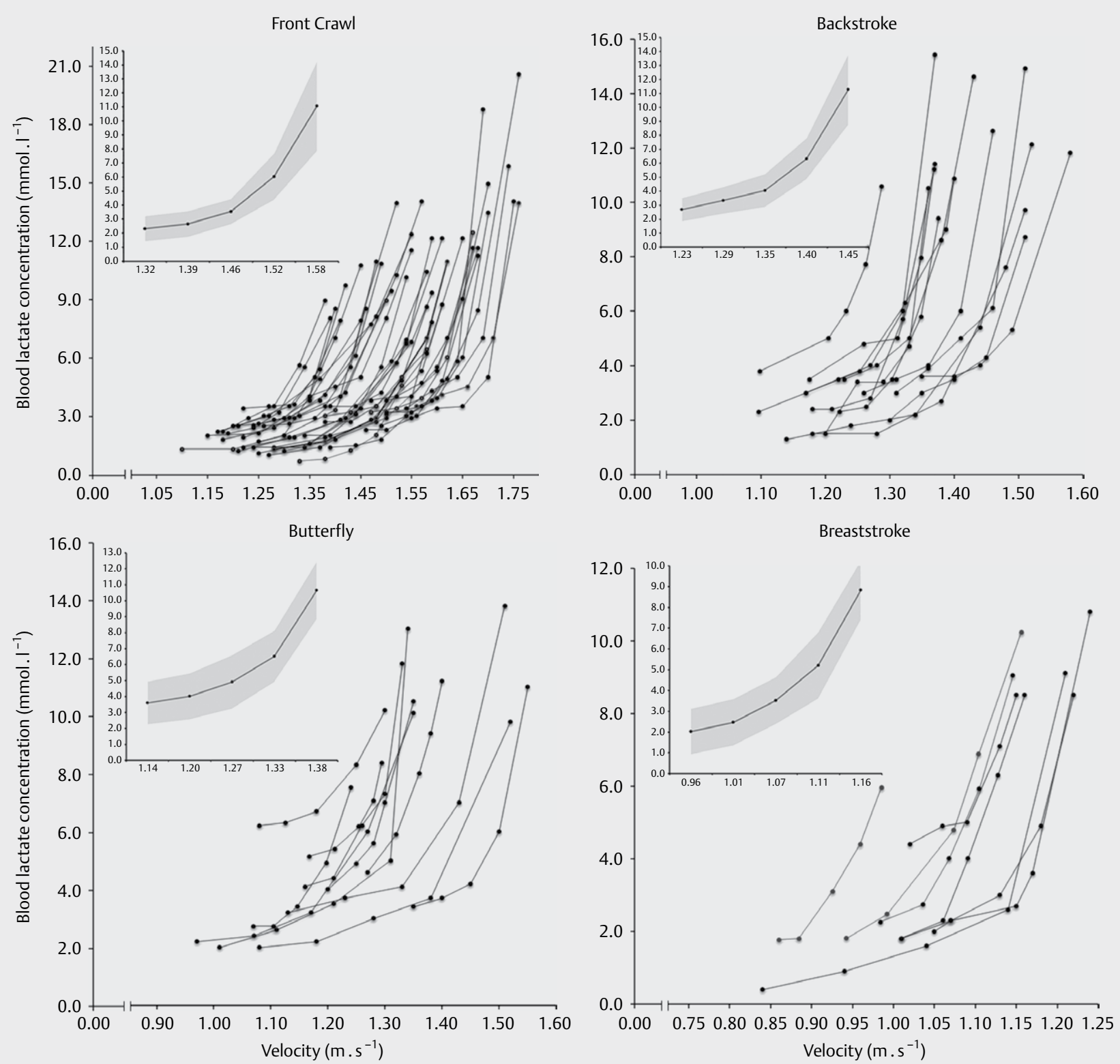

- Fig. 2 Individual and mean \pm standard deviation envelope blood lactate concentrations vs velocity curves for individual anaerobic threshold assessment in each conventional swimming technique.

front crawl presents higher BLIS in the $4^{\text {th }}$ and $5^{\text {th }}$ step compared to breaststroke and butterfly, but similar compared to backstroke, and (iv) alternated swimming techniques present higher BLIS than simultaneous ones. Combining BLIS with the AnT assessment in the incremental protocol is very relevant allowing researchers and coaches to evaluate both aerobic and anaerobic behaviors in a single test session.

\section{Discussion}

One of the main purposes of AnT assessment is to evaluate the adequacy of aerobic training. As its development is one of the most important targets in competitive swimming, we assessed the AnT through a biophysical approach for all four conventional swimming techniques. Despite a variety of different AnT testing procedures in swimming, the majority are not regularly used, since: (i) the noninvasive $30 \mathrm{~min}$ and $2000 \mathrm{~m}$ tests $[7,25]$ are monotonous and not motivational, possibly leading to an underestimation of the final result; (ii) the maximal lactate steady state test $[10,26]$, although still considered the gold standard for assessing aerobic capacity requires, at least, 3 consecutive days of testing; (iii) the critical velocity test, composed by, at least, two maximal intensity bouts, can lead to an overestimation of the final result if a distance of $\sim 15 \mathrm{~min}$ is not used [27]; (iv) the long duration tests probably are not performed at constant velocities, reflecting different intensities levels, and are difficult to apply using simultaneous swimming techniques 

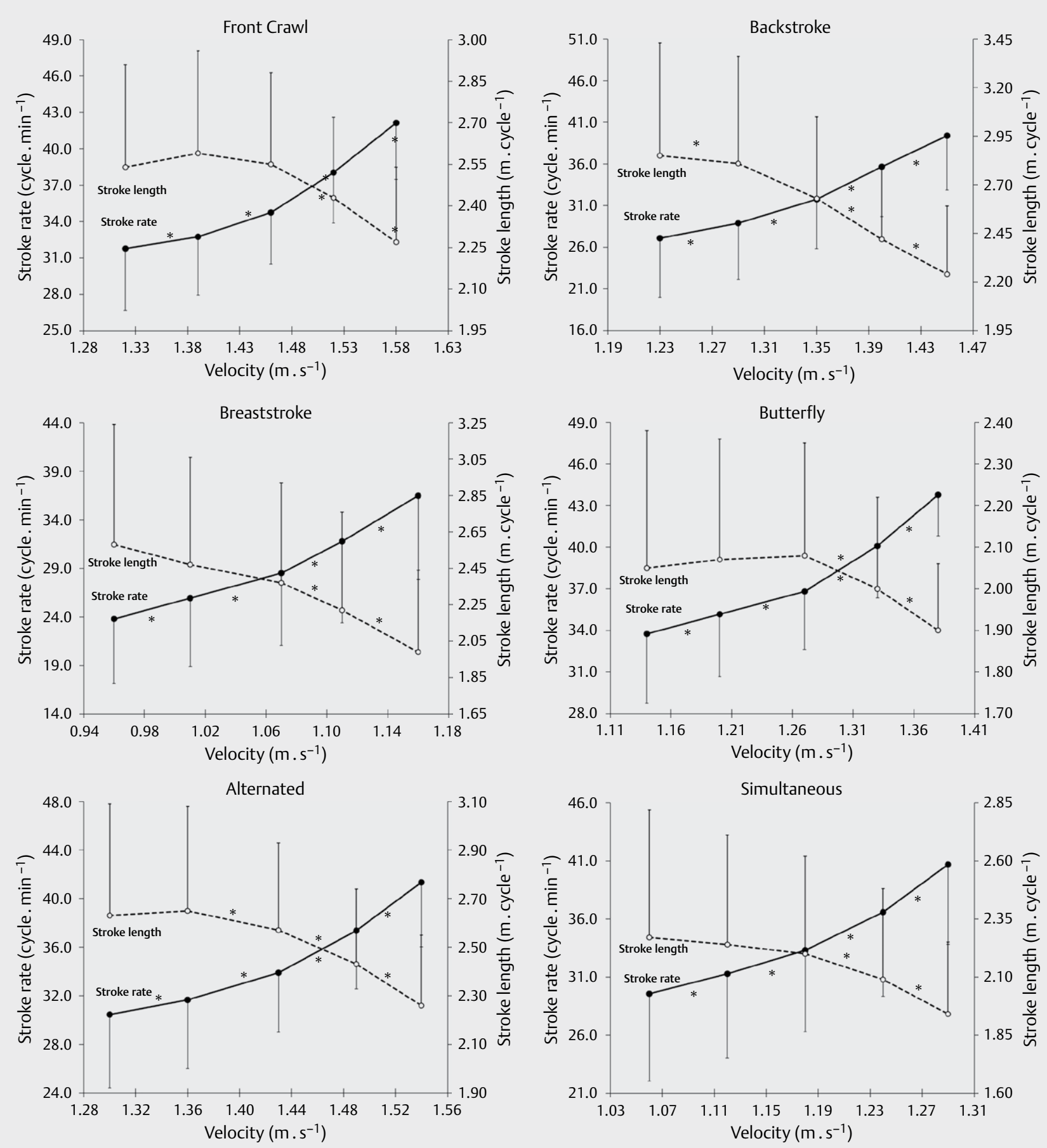

- Fig. 3 Mean and standard deviations for stroke rate and length along the incremental protocol for assessment of individual anaerobic threshold for each competitive swimming techniques and by alternated and simultaneous upper and lower limb-action groups.

[28]; (v) the two speed test has the limitation inherent to the [La] $4 \mathrm{mmol} \cdot \mathrm{I}^{-1}$ average value $[14,20]$.

To overcome the above referred constrains, we have adapted one of the most used swimming intermittent incremental protocols $[8,29,30]$ to assess the AnT in the four conventional swimming techniques. This $5 \times 200 \mathrm{~m}$ incremental protocol was already used for front crawl $[3,20]$ and appears to be a useful procedure to de- termine the exact exercise intensity corresponding to the beginning of an exponential [La] rise in the four competitive swimming techniques. In addition, as it is shorter in duration comparing to other incremental protocols (e.g., [8]), it might be more frequently implemented in swimming training cycles, particularly at elite level, allowing a more regular and systematic training control and swimmers evaluation. 
- Table 1 Mean and SD values of all assessed variables in the $5 \times 200 \mathrm{~m}$ incremental protocol.

\begin{tabular}{|c|c|c|c|c|c|c|}
\hline Variables & $\begin{array}{l}\text { Front crawl } \\
(\mathrm{n}=38)\end{array}$ & $\begin{array}{l}\text { Backstroke } \\
(n=15)\end{array}$ & $\begin{array}{l}\text { Breaststroke } \\
(n=8)\end{array}$ & $\begin{array}{l}\text { Butterfly } \\
(n=11)\end{array}$ & $\begin{array}{l}\text { Alternated } \\
\text { techniques }(n=53)\end{array}$ & $\begin{array}{l}\text { Simultaneous } \\
\text { techniques }(n=19)\end{array}$ \\
\hline$[\mathrm{La}]_{\mathrm{AnT}}\left(\mathrm{mmol} \cdot \mathrm{I}^{-1}\right)$ & $3.3 \pm 1.0^{+}$ & $3.9 \pm 1.1$ & $2.9 \pm 1.1^{+}$ & $4.9 \pm 1.5$ & $3.4 \pm 1.1$ & $4.0 \pm 1.7$ \\
\hline$v_{\text {AnT }}\left(m \cdot s^{-1}\right)$ & $1.45 \pm 0.13^{+,++}$ & $1.35 \pm 0.09^{++}$ & $1.06 \pm 0.09$ & $1.29 \pm 0.10^{++}$ & $1.43 \pm 0.13$ & $1.19 \pm 0.15$ \\
\hline$v 4\left(m \cdot s^{-1}\right)$ & $1.48 \pm 0.10^{*}$ & $1.35 \pm 0.12$ & $1.08 \pm 0.08$ & $1.22 \pm 0.16$ & $1.44 \pm 0.12 *$ & $1.16 \pm 0.15$ \\
\hline $\mathrm{v} 3.5\left(\mathrm{~m} \cdot \mathrm{s}^{-1}\right)$ & $1.45 \pm 0.10$ & $1.30 \pm 0.12^{*}$ & $1.07 \pm 0.09$ & $1.17 \pm 0.17^{*}$ & $1.41 \pm 0.12^{*}$ & $1.13 \pm 0.15^{*}$ \\
\hline $\mathrm{vSR}_{\text {inf }}\left(\mathrm{m} \cdot \mathrm{s}^{-1}\right)$ & $1.45 \pm 0.13$ & $1.35 \pm 0.10$ & $1.07 \pm 0.07$ & $1.28 \pm 0.08$ & $1.42 \pm 0.13$ & $1.19 \pm 0.13$ \\
\hline$v S L_{\text {inf }}\left(m \cdot s^{-1}\right)$ & $1.45 \pm 0.13$ & $1.35 \pm 0.10$ & $1.07 \pm 0.08$ & $1.28 \pm 0.08$ & $1.42 \pm 0.13$ & $1.20 \pm 0.13$ \\
\hline$[\mathrm{La}]_{\text {rate }}\left[\left(\mathrm{mmol} \cdot \mathrm{l}^{-1}\right) \cdot\left(\mathrm{m} \cdot \mathrm{s}^{-1}\right)\right]$ & $93.5 \pm 44.2$ & $118.7 \pm 49.7$ & $71.8 \pm 11.6$ & $89.5 \pm 69.8$ & $99.6 \pm 46.3$ & $82.4 \pm 54.0$ \\
\hline $4^{\text {th }}$ BLIS $\left[\left(\mathrm{mmol} \cdot \mathrm{I}^{-1}\right) \cdot \mathrm{s}^{-1}\right]$ & $0.02 \pm 0.01^{+,++}$ & $0.02 \pm 0.01$ & $0.01 \pm 0.00$ & $0.01 \pm 0.01$ & $0.02 \pm 0.01^{\#}$ & $0.01 \pm 0.00$ \\
\hline $5^{\text {th }}$ BLIS $\left[\left(\mathrm{mmol} \cdot \mathrm{l}^{-1}\right) \cdot \mathrm{s}^{-1}\right]$ & $0.04 \pm 0.02^{+,++, \# \#}$ & $0.04 \pm 0.02^{\# \#}$ & $0.02 \pm 0.01^{\# \#}$ & $0.03 \pm 0.02^{\# \#}$ & $0.04 \pm 0.02^{\#, \# \#}$ & $0.03 \pm 0.02^{\# \#}$ \\
\hline
\end{tabular}

Abbreviations: [La] $]_{A n T}$, blood lactate concentration at individual anaerobic threshold; $\mathrm{v}_{\mathrm{An}}$, $\mathrm{v} 4$ and $\mathrm{v} 3.5$, velocities corresponding to individual anaerobic threshold, 4 and $3.5 \mathrm{mmol} \cdot \mathrm{I}^{-1}$ of blood lactate concentrations; $v \mathrm{SR}_{\text {inf }}$ and vSL $\mathrm{L}_{\text {inf }}$, velocities corresponding to stroke rate a length inflexion points; [La $]_{\text {rate }}$ blood lactate increase rate between last two steps of the protocol; $4^{\text {th }}$ and $5^{\text {th }}$ BLIS, blood lactate increase speed of $4^{\text {th }}$ and $5^{\text {th }}$ steps. ${ }^{*,+,++, \#, \# \#}$ differences from $v_{A n T}$, butterfly, breaststroke, simultaneous techniques and $4^{\text {th }} B L I S$, respectively $(p \leq 0.05)$.

In front crawl, [La $]_{\text {AnT }}$ was lower than the fixed $4 \mathrm{mmol} \cdot \mathrm{I}^{-1}$ value, in accordance with the literature $[10,17,20]$, but similar to the $3.5 \mathrm{mmol} \cdot \mathrm{I}^{-1}$ value proposed by Heck et al. (1985) for highly aerobically trained athletes. This reinforces the idea that $\mathrm{v}_{4}$ does not represent the $v_{A n T}$ and should be used with caution for training prescription. Backstroke and butterfly $\mathrm{v}_{\mathrm{AnT}}$ were similar to $\mathrm{v}_{4}$ and higher than $v_{3.5}$, but no differences were observed for breaststroke (probably due to small sample size). After the[La] evidencing an exponential behaviour, a small increase in swimming velocity will imply a high increment in [La], justifying the similarity between the $\mathrm{v}_{\mathrm{AnT}}, \mathrm{v}_{4}$ and $\mathrm{v} 3.5$ values, even with $[\mathrm{La}]_{\mathrm{AnT}}$ lower than $3.5 \mathrm{mmol} \cdot \mathrm{I}^{-1}$.

In fact, the mean [La $]_{A n T}$ values differ between swimming techniques, with a range from $<2$ to $>5 \mathrm{mmol} \cdot \mathrm{l}^{-1}$, showing a high inter-variability. This may be justified by the high specialized training process of elite swimmers (in advanced moments of their sporting career the training load is increasingly anaerobic), particularly for non-front crawl specialists, as their competitive events lasts from 50 to $200 \mathrm{~m}$ in opposition to the typical non-oriented and high-volume training [2]. This underlines the importance of determining the individual AnT instead of using the 4 or $3.5 \mathrm{mmol} \cdot \mathrm{I}^{-1}$ averaged values that do not take into consideration the inter-individual variability or technique and event specificity $[7,14,20]$.

The higher $[\mathrm{La}]_{\mathrm{AnT}}$ found for butterfly compared to front crawl and backstroke was expected, since the higher butterfly intracycle velocity variations leads to an increased energy cost and anaerobic contribution to reach the energy demands of that specific swimming technique $[18,31]$. However, the lower [La $]_{\text {AnT }}$ in breaststroke comparing to backstroke was not anticipated, conflicting with our previous hypothesis. As the $v_{\text {AnT }}$ in breaststroke was also considerably lower than all other swimming techniques, the possible lower energy output requirement of this simultaneous swimming technique $[18,32]$ could justify these results.

The above-referenced comparisons between swimming techniques were made possible since $v_{\text {AnT }}$ occurred at $~ 92 \%$ of the velocity of the last protocol step in all four of them, a similar value to that found in the literature $[33,34]$. However, [La $]_{\text {AnT }}$ assessment were not reliable when analysing swimming techniques between upper and lower limb-action groups, since a [La $]_{\text {AnT }}$ lower than $3.5 \mathrm{mmol} \cdot \mathrm{I}^{-1}$ corresponded to a higher velocity than $\mathrm{v} 3.5$, confirming the assertion that swimming techniques are not equal biophysical terms [4, 18, 31]. Moreover, we observed that front crawl presented higher velocities in all five steps of the incremental protocol. Therefore, AnT assessment should be undertaken in each swimmer's specialist technique.

Regarding the general biomechanical parameters, low SR and high SL values were constant within the steps swum at low to moderate intensities (below and at the AnT), in which the aerobic system is predominant (for all techniques) $[8,15,17]$. Above the AnT, at heavy and severe intensities $[11,12]$, where the anaerobic contributions start to increase, SR and SL showed an opposite behaviour in response to the metabolic imbalance $[10,11,17]$ and an increased hydrodynamic drag $[35,36]$. These changes were not strictly linear and a critical turning point was identified for both physiological and biomechanical variables, in accordance with front crawl literature $[10,11,17]$. In some cases, SL presented an inverted "U shape” justifying the close values between the first and last step. However, the same SL behaviour after AnT was evident for all swimmers. Good agreement was also observed between $\mathrm{v}_{\mathrm{AnT}}$, $\mathrm{VSR}_{\text {inf }}$ and $v \mathrm{VL}_{\text {inf }}$, corroborating the above-referenced previous findings.

Since most competitive swimming events have a short duration (from 20 s to $5 \mathrm{~min}$ ), [La] rate and BLIS were determined to complement the AnT assessment to explore the anaerobic capability of highly trained swimmers. [La] rate assessment was adapted from Holroyd \& Swanwick (1993) lactate tolerance rating (differential velocity between [La] of 5.0 and $10.0 \mathrm{mmol} \cdot \mathrm{I}^{-1}$ ) since that methodology could not be used in our entire sample because some swimmers have their AnT higher $5 \mathrm{mmol} \cdot \mathrm{I}^{-1}$ and others presented values lower $10 \mathrm{mmol} \cdot \mathrm{I}^{-1}$ in the last step of the incremental protocol. No differences were observed in [La $]_{\text {rate }}$ between swimming techniques, probably related to the non-regular velocity differential between the last two steps of the incremental protocol (the last $200 \mathrm{~m}$ were done at maximal effort). However, the use of the $[\mathrm{La}]_{\text {rate }}$ in consecutive evaluations may be useful for identification of swimmers strengths and weakness regarding the use of anaerobic energy. 

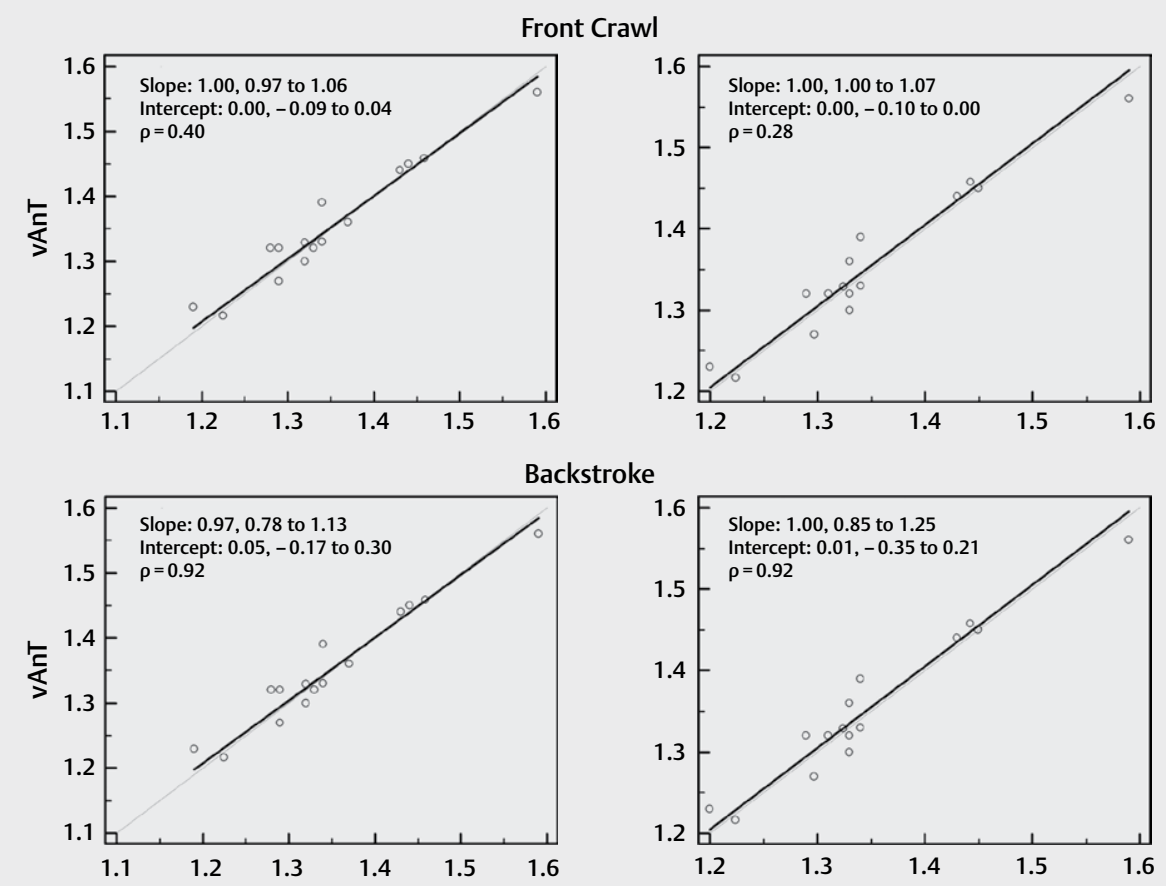

Breaststroke
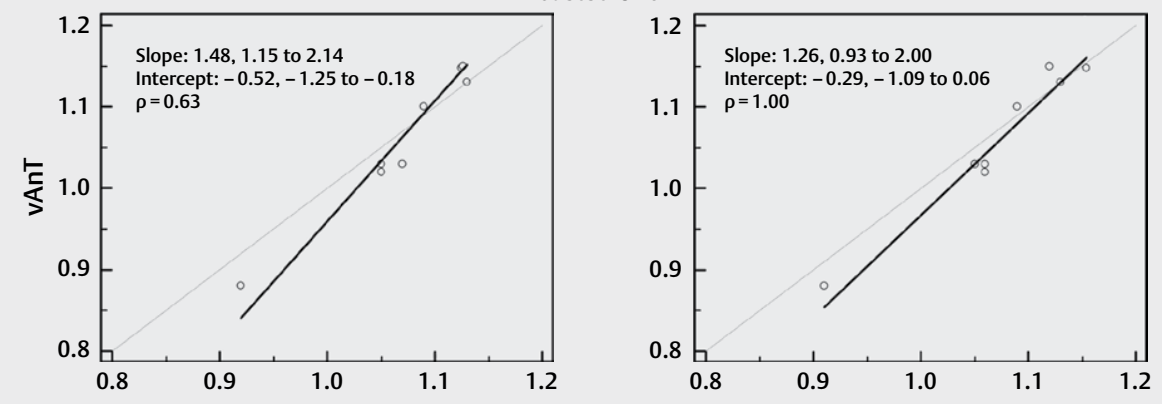

Butterfly
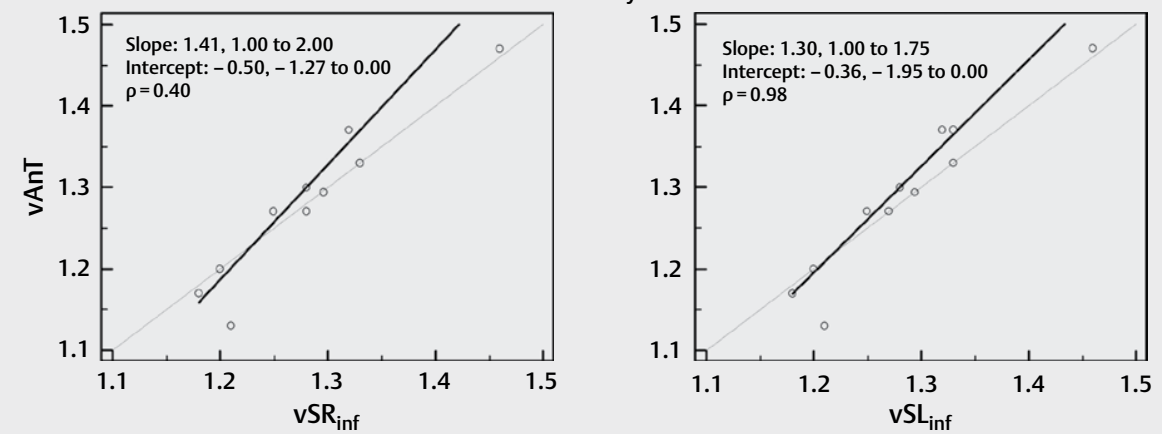

- Fig. 4 Passing-Bablok regression of velocities at individual anaerobic threshold and at stroke rate inflexion points (left panels), and stroke length (right panels). The regression equation and the identity are represented by the solid and dotted lines, respectively. Regression equation ( $y=a x+b)$ evidences existence of proportional (slope: a) and systematic (intercept: b) differences and respective $95 \%$ confidence intervals ( $\mathrm{Cls}$ ). If zero is in the $95 \% \mathrm{Cls}$ of the intercept, and one in the $95 \% \mathrm{Cls}$ of the slope, the two methods are comparable. If zero is not in the $95 \% \mathrm{Cls}$ of the intercept there is a systematic difference and if one is not in the $95 \% \mathrm{Cls}$ of the slope exists a proportional difference between the two methods. Passing-Bablok method deemed applicable if the relationship between the two measurements is linear $(\rho>0.05)$. Abbreviations: vAnT, velocity corresponding to individual anaerobic threshold; $v S R_{\text {inf }}$ and $v S L_{\text {inf }}$, velocities corresponding to stroke rate and length inflexion points.

Higher anaerobic contribution at the end of the incremental protocol was indicated by a higher BLIS of the $5^{\text {th }}$ step than the $4^{\text {th }} 200 \mathrm{~m}$ stage (as the last step was conducted at maximal exertion) [17]. An- other contributing evidence was the fact that swimmers with a higher SR, at the final of the incremental protocol, could not maintain their SL $[12,15]$. The higher BLIS values observed on the alter- 

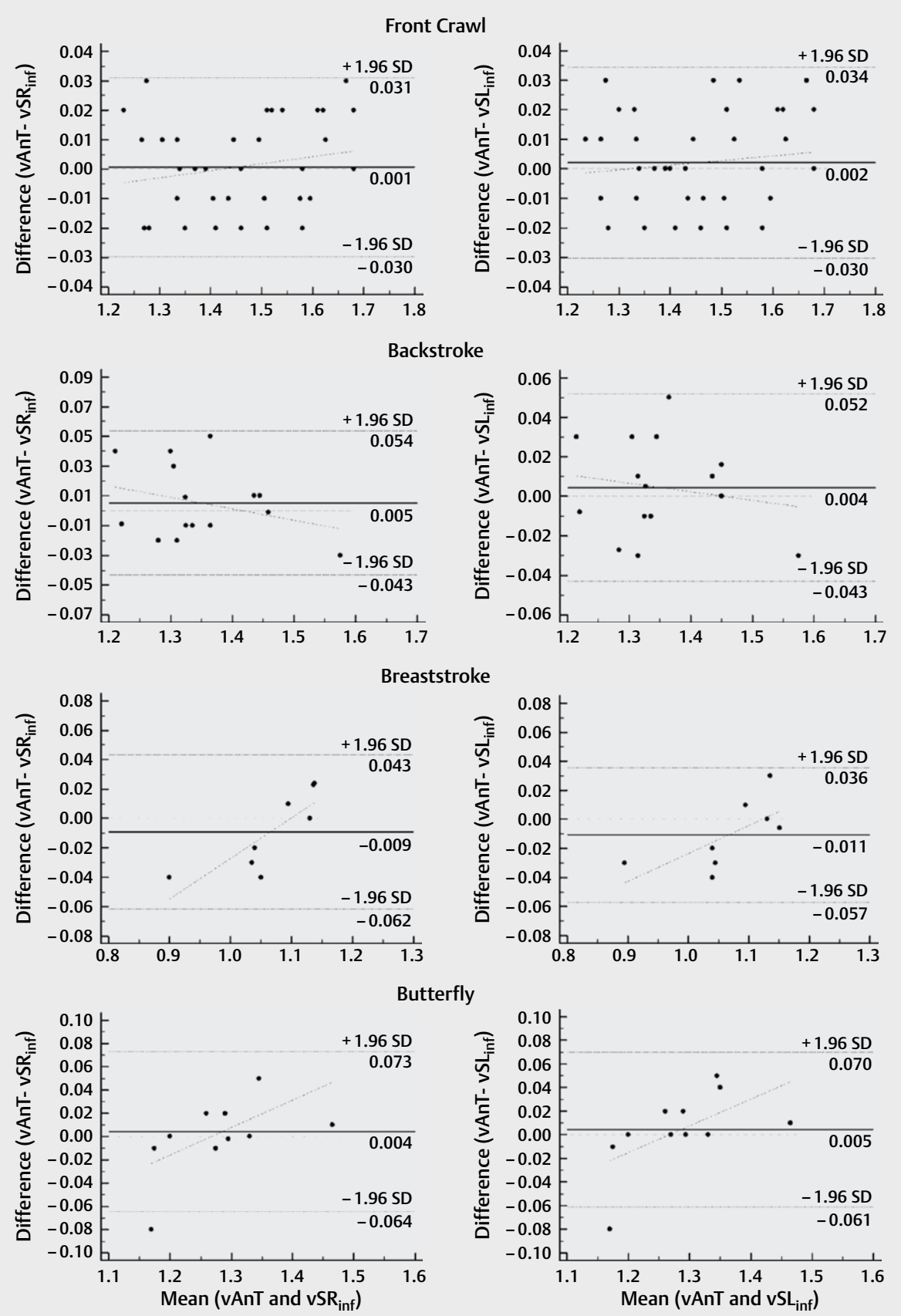

- Fig. 5 Bland Altman analyses between velocity corresponding to individual anaerobic threshold and velocity of stroke rate (left panels), and stroke length (right panels) inflection points. Black dotted lines representing 95\% limits of agreement and black dashed lines bias and linear regression of physiological and biomechanical variables. Abbreviations: vAnT, velocity corresponding to individual anaerobic threshold; $\mathrm{VSR}_{\text {inf }}$ and vSL $\mathrm{L}_{\text {inf }}$, velocities corresponding to stroke rate and length inflexion points.

nated swimming techniques can be justified by the higher velocities achieved at the $4^{\text {th }}$ and $5^{\text {th }}$ step (comparing to simultaneous techniques) and consequent increased metabolic demands. Curiously, BLIS of the last butterfly step was lower than a previous study using an all-out $200 \mathrm{~m}$ butterfly effort [37], evidencing the effect of previous exercise on the energetic pathways behaviour during the incremental protocol (indicating a progressive decrease in glycolytic power). These data may also indicate that the aerobic system is potentiated at submaximal exertions, allowing a higher blood lactate metabolisation during exercise at maximal effort [38], particularly during the last step of the $5 \times 200 \mathrm{~m}$ intermittent incremental protocol. 


\section{Conclusion}

The widely used $5 \times 200 \mathrm{~m}$ intermittent incremental protocol allowed a specific and precise AnT assessment in the four conventional swimming techniques. Although it appears that the $3.5 \mathrm{mmol} \cdot \mathrm{I}^{-1}$ threshold value (rather than $4.0 \mathrm{mmol} / \mathrm{l}^{-1}$ ) is more suitable for front crawl, an individual assessment is the better option. It was also possible to establish the AnT as a biophysical boundary for all competitive swimming techniques, allowing very practical information for coaches. Although it may be easier to determine the AnT using biomechanical variables, it is important to initially analyse the swimmers' AnT through a physiological approach, to determine whether the difference between the two approaches is significant in actual practice. The BLIS indicator was useful for assessing swimmers anaerobic behaviour and training exercises above AnT.

\section{Funding}

This work is supported by national funding through the Portuguese Foundation for Science and Technology, I.P., under CIFI2D PhD individual grant: SFRH/BD/138876/2018 and project CIDESD: UID/ DTP/04045/2019.

\section{Conflict of Interest}

Authors declare that they have no conflict of interest.

\section{References}

[1] Almeida-Coelho J, Fernandes RJ, Vilas-Boas JP. Metabolic and technical changes in swimmers during a 100-m all-out front crawl. Trends Sport Sci 2016; 23: 177-183

[2] Nugent F], Comyns TM, Burrows E et al. Effects of low-volume, high-intensity training on performance in competitive swimmers: A systematic review. J Strength Cond Res 2017; 31: 837-847

[3] Thanopoulos V. The $5 \times 200 \mathrm{~m}$ step test lactate curve model: gender specific characteristics in elite greek senior freestyle swimmers. Serb J Sports Sci 2010; 4: 153-160

[4] Gonjo T, McCabe C, Sousa A et al. Differences in kinematics and energy cost between front crawl and backstroke below the anaerobic threshold. Eur J Appl Physiol 2018; 118: 1107-1118

[5] Mader A, Heck H, Hollmann W. Evaluation of latic acid anaerobic energy contribution by determination of post exercise latic acid concentration of ear capillary blood in middle-distance runners and swimmers. In: Landry F, Orban W, Eds. Exercise Physiology. Miami: Symposia Specialists; 1978: 187-200

[6] Wasserman K, Whipp B], Koyl SN et al. Anaerobic threshold and respiratory gas exchange during exercise. J Appl Physiol 1973; 35: 236-243

[7] Faude O, Kindermann W, Meyer T. Lactate thresholds concepts. How valid are they? Sports Med 2009; 39: 469-490

[8] Fernandes RJ, Sousa M, Machado L et al. Step length and individual anaerobic threshold assessment in swimming. Int J Sports Med 2011; 32: $940-946$

[9] Olbrecht ], Madsen O, Mader A et al. Relationship between swimming velocity and lactic concentration during continuous and intermittent training exercises. Int J Sports Med 1985; 6: 74-77
[10] Pelarigo JG, Greco CC, Denadai BS et al. Do $5 \%$ changes around maximal lactate steady state lead to swimming biophysical modifications? Hum Mov Sci 2016; 49: 258-266

[11] de Jesus K, Sanders R, de Jesus K et al. The effect of intensity on 3-dimensional kinematics and coordination in front-crawl swimming. Int J Sports Physiol Perform 2016; 11: 768-775

[12] Ribeiro J, Toubekis AG, Figueiredo P et al. Biophysical determinants of front-crawl swimming at moderate and severe Intensities. Int J Sports Physiol Perform 2017; 12: 241-246

[13] Sousa A, Figueiredo P, Zamparo P et al. Exercise modality effect on bioenergetical performance at $\dot{V}_{2}$ max intensity. Med Sci Sports Exerc 2015; 47: 1705-1713

[14] Stegmann H, Kindermann W. Comparison of prolonged exercise tests at the individual anaerobic threshold and the fixed anaerobic threshold of 4 mmol - L-1 lactate. Int J Sports Med 1982; 3: 105-110

[15] Barbosa TM, Fernandes RJ, Keskinen KL et al. The influence of stroke mechanics into energy cost of elite swimmers. Eur J Appl Physiol 2008; 103: 139-149

[16] Keskinen KL, Komi PV. Stroking characteristics of front crawl swimming during exercise. J Appl Biomech 1993; 9: 219-226

[17] Figueiredo P, Morais P, Vilas-Boas JP et al. Changes in arm coordination and stroke parameters on transition through the lactate threshold. Eur J Appl Physiol 2013; 113: 1957-1964. doi:10.1007/s00421-013-2617-8

[18] Barbosa TM, Fernandes RJ, Keskinen KL et al. Evaluation of the energy expenditure in competitive swimming strokes. Int J Sports Med 2006; 27: 894-899

[19] Harriss DJ, Macsween A, Atkinson G. Standards for ethics in sport and exercise science research: 2020 update. Int J Sports Med 2019; 40: 813-817

[20] Fernandes RJ, Sousa M, Pinheiro A et al. Assessment of individual anaerobic threshold and stroking parameters in swimmers aged 10-11 years. Eur J Sport Sci 2010; 10: 311-317

[21] Holroyd A, Swanwick K. A mathematical model for lactate profiles and a swimming power expenditure formula for use in conjunction with it. J Swim Res 1993; 9: 25-31

[22] Hopkins WG. A Scale of Magnitudes for Effect Statistics. A new view of statistics 2002.“On de internet: https://www.sportsci.org/resource/ stats/effectmag.html” Accessed in 9 November 2018

[23] Passing $\mathrm{H}$, Bablok W. A new biometrical procedure for testing the equality of measurements from two different analytical methods. Application of linear regression procedures for method comparison studies in clinical chemistry. Part I. J Clin Chem Clin Biochem 1983; 21: 709-720

[24] Bland JM, Altman DG. Statistical methods for assessing agreement between two methods of clinical measurement. Lancet 1986; 1: 307-310

[25] Pine DB, Touretski G. An analysis of the training of Olympic sprint champion Alexandre Popov. Australian Swim Coach 1993; 10: 5-14

[26] Heck H, Mader A, Hess G et al. Justification of the 4-mmol/l lactate threshold. Int J Sports Med 1985; 6: 117-130

[27] Wright B, Smith D]. A protocol for the determination of critical speed as an index of swimming endurance performance. In: Miyashita M, Mutoh Y, Richardson AB, Eds. Medicine and Science in Aquatic Sports, Med Sport Sci, Karger, Basel; 1994: 39: 55-59

[28] Smith DJ, Norris SR, Hogg JM. Performance evaluation of swimmers. Scientific tools. Sports Med 2002; 32: 539-554

[29] Anderson M, Hopkins W, Roberts A et al. Ability of test measures to predict competitive performance in elite swimmers. J Sports Sci 2008; 26: $123-130$

[30] Pyne DB, Lee H, Swanwick KM. Monitoring the lactate threshold in world-ranked swimmers. Med Sci Sports Exerc 2001; 33: 291-297 
[31] Vilas-Boas JP, Fernandes RJ, Barbosa TM. Intra-cycle velocity variations, swimming economy, performance and training in swimming. In: Seifert L, Chollet D, Mujika I, Eds. The World Book of Swimming: From Science to Performance. New York: Nova Science Publishers; 2011: 119-134

[32] Hellard P, Pla R, Rodríguez FA et al. Dynamics of the metabolic response during a competitive $100-\mathrm{m}$ freestyle in elite male swimmers. Int J Sports Physiol Perform 2018; 1-28

[33] Fernandes R], Keskinen KL, Colaço P et al. Time limit at $\dot{\mathrm{VO}}_{2} \max$ velocity in elite crawl swimmers. Int J Sports Med 2008; 29: 145-150

[34] Zacca R, RJP Fernandes, Pyne DB et al. Swimming training assessment: the critical velocity and the 400 -m test for age-group swimmers. J Strength Cond Res 2016; 30: 1365-1372
[35] Komar ], Leprêtre PM, Alberty M et al. Effect of increasing energy cost on arm coordination in elite sprint swimmers. Hum Mov Sci 2012; 31 : 620-629

[36] Seifert L, Komar ], Crettenand F et al. Inter-limb coordination and energy cost in swimming. J Sci Med Sport 2014; 17: 439-444

[37] Ferreira M, Figueiras T, Vilas-Boas JP et al. Blood lactate and ammonia kinetics along the $200 \mathrm{~m}$ butterfly swimming event: Comparison among adult and juvenile swimmers. In: Eriksson BO, Gullstrand L, Eds. XII FINA World Congress on Sports Medicine. Göteborg: FINA, 1997; 298-303

[38] Brooks GA. Cell-cell and intracellular lactate shuttles. J Physiol 2009; 587: 5591-5600 